\title{
Quality of life, depression, anxiety and loneliness in patients with bullous pemphigoid. A case control study ${ }^{*}$
}

\author{
Anargyros Kouris ${ }^{1}$ \\ Christos Christodoulou ${ }^{2}$ \\ Panagiota Korkoliakou ${ }^{2}$ \\ Revekka Tsatovidou ${ }^{2}$ \\ George Kontochristopoulos ${ }^{1}$
}

\author{
Eftychia Platsidaki ${ }^{1}$ \\ Kalliopi Armyra ${ }^{1}$ \\ Christina Stefanaki ${ }^{1}$ \\ Dimitrios Rigopoulos 2
}

DOI: http:/ / dx.doi.org/10.1590/abd1806-4841.20164935

\begin{abstract}
BACKGROUND: Bullous pemphigoid (BP) is a chronic, autoimmune blistering skin disease that affects patients' daily life and psychosocial well-being.

ОвјестіVе: The aim of the study was to evaluate the quality of life, anxiety, depression and loneliness in BP patients.

MethoDs: Fifty-seven BP patients and fifty-seven healthy controls were recruited for the study. The quality of life of each patient was assessed using the Dermatology Life Quality Index (DLQI) scale. Moreover, they were evaluated for anxiety and depression according to the Hospital Anxiety Depression Scale (HADS-scale), while loneliness was measured through the Loneliness Scale-Version 3 (UCLA) scale.

RESULTS: The mean DLQI score was 9.45 \pm 3.34 . Statistically significant differences on the HADS total scale and in HADS-depression subscale ( $p=0.015$ and $p=0.002$, respectively) were documented. No statistically significant difference was found between the two groups on the HADS-anxiety subscale. Furthermore, significantly higher scores were recorded on the UCLA Scale compared with healthy volunteers $(\mathrm{p}=0.003)$.

CONCLUSION: BP had a significant impact on quality of life and the psychological status of patients, probably due to the appearance of unattractive lesions on the skin, functional problems and disease chronicity.
\end{abstract}

Keywords: Anxiety; Depression; Loneliness; Pemphigoid, bullous; Quality of life

\section{INTRODUCTION}

Bullous pemphigoid (BP) is a blistering disease of the skin, usually affecting middle-aged or elderly persons. It is characterized by an intensely pruritic rash, dominated by tense blisters arising in normal looking or erythematous skin. It may be localized to one area, or widespread on the trunk and proximal limbs. ${ }^{1-3} \mathrm{BP}$ is likely to have psychological and social implications for the patient since it is a chronic condition. Both clinical symptoms and the effects of treatment can have a strong impact on physical and emotional status. ${ }^{4,5}$ They may lead to functional limitations, a need for increased family support, stress and exclusion from social activities. There are few reports in the literature regarding $\mathrm{BP}^{\prime}$ s impact on patients' qual- ity of life but these are frequently underestimated by clinicians. ${ }^{4,6}$ The aim of the study was to evaluate the quality of life, anxiety, depression and loneliness in BP patients.

\section{MATERIALS}

We enrolled patients referred to the "Andreas Sygros" Skin Hospital (tertiary referral center) due to BP. The study protocol was approved by the Ethics Committee of the "Andreas Sygros" Hospital and written informed consent was obtained from all patients. The diagnosis was based on clinical and histological grounds. Patients considered eligible for inclusion were aged over 18 years, able to

Received on 16.07.2015.

Approved by the Advisory Board and accepted for publication on 10.10.2015.

* Work performed at the Department of Dermatology and Venereology, "Andreas Sygros" Skin Hospital and Department of Psychiatry, "Attikon" University Hospital - Athens, Greece.

Financial Support: None.

Conflict of Interest: None.

"Andreas Sygros" Skin Hospital - Athens, Greece.

"Attikon" University Hospital - Athens, Greece.

C2016 by Anais Brasileiros de Dermatologia 
understand the Greek language and had not received any treatment during the previous three months. The study excluded patients with a psychiatric history or a history of psychotropic drug use.

To assess patients' quality of life, the Dermatology Life Quality Index (DLQI) was used.7 They were also evaluated for anxiety and depression, drawing on the Hospital Anxiety and Depression Scale (HADS), while loneliness was measured by the UCLA loneliness Scale (UCLA-Version 3). ${ }^{8-11}$

The DLQI is a validated, 10-item questionnaire on personal relationships, leisure, daily activities and treatment. The maximum score is 30 , with 0 indicating the least impairment and 30 the greatest impairment in the patient's quality of life. ${ }^{7}$

The Hospital Anxiety and Depression Scale (HADS) developed by Zigmond and Snaithand constitutes an instrument for detecting states of anxiety and depression originally of hospital medical outpatients. It is also appropriate for medical patients in general, as it does not refer to the somatic symptoms of anxiety and depression. ${ }^{8}$ The HADS is a self-report rating scale of 14 items designed to measure anxiety and depression, the emotions that most commonly cause psychological distress in patients (seven items for each subscale). Responses to items are indicated on a four-point Likert Scale, ranging from 0 to 3 (score range $0-21$, for each subscale), where high scores indicate more symptoms. The authors of the previous study suggested two cut-off scores for detecting depression and anxiety that have commonly been used in most studies; scores of 8 to 10 denote doubtful cases, while scores of 11 and above signify valid cases. HADS has been validated in the Greek population'. In this study and in the outpatient group, HADS values were estimated as follows: total HADS 16.1 \pm 7.4 , HADS-Anxiety $8.7 \pm 4.3$ and HADS-Depression $7.3 \pm 3.8$; whereas, in the general population group, they were: $9.1 \pm 6.1,5.1 \pm 3.7$ and $3.9 \pm 3.1$, respectively. ${ }^{9}$

Additionally, patients were assessed for loneliness according to the UCLA scale (Version 3). It is a 20-item questionnaire, which measures personal perception of loneliness and social isolation; higher UCLA scores indicate stronger feelings of loneliness and isolation. The UCLA scale has also been validated in the Greek population $^{11}$. In the general population, UCLA-loneliness scale values were estimated to be $40.08 \pm 9.50$ for young individuals and $31.51 \pm 6.92$ for the elderly ${ }^{10}$.

The control group was recruited during the same period and included fifty-seven healthy, age- and sex-matched volunteers from the general population (in our study, individuals from the administrative staff of the hospital were randomly selected), with no personal history of BP or any history of psychotropic drug use. Participation in the control group was voluntary and without financial compensation.

\section{Statistical analysis}

All data were analyzed using the statistical package for social science (SPSS 17.0) for Windows. The t-test was used for quantitative analyses and comparison of means, the chi- square test was employed for qualitative analyses, while the Pearson's coefficient correlation (r) was also applied. Results were expressed as means +/-SD. The statistical significance was determined as a $p$ value of 0.05 .

\section{RESULTS}

This case-control study included 57 patients, of whom 31 were female and 26 were male, aged $39-79$ (mean $59.73 \pm 10.77$ ). The control group consisted of an equal number of healthy individuals (33 females and 24 males), aged 38-81 (mean 60.66 \pm 11.26 ). No statistically significant (NS) difference between patients and controls was documented in terms of age $(t=0.45, p>0.05, N S)$ and sex $\left(x^{2}=0.14\right.$, $\mathrm{p}>0.05, \mathrm{NS})$.

The DLQI score was $9.45 \pm 3.34$ in BP patients. Significantly higher scores were recorded on the UCLA Scale (43.01 \pm 5.66$)$ compared with volunteers $(39.12 \pm 7.79)(p=0.003)$. Regarding the subscale HADS-depression scores and HADS-total scale, BP patients had statistically significant higher scores compared with healthy controls ( $p=0.002$ and $p=0.015$, respectively). In contrast, no statistically significant difference was found between the two groups for subscale HADS-anxiety (Table 1). The duration of symptoms ranged from three weeks to nine months (average duration was two months). A positive correlation was noted between loneliness and depression $(\mathrm{r}=0.216, \mathrm{p}=0.021)$; and anxiety and depression $(\mathrm{r}=0.489$, $\mathrm{p}<0.0001)$. No correlation was noted between the DLQI scores and anxiety, depression, loneliness or age. Similarly, no correlation was observed between UCLA scale and age, anxiety, total score of HADscale and DLQI scores.

\section{DISCUSSION}

$\mathrm{BP}$ can be a distressing disease and it represents a lifelong burden for affected patients. Our data showed that the mean DLQI score was $9.45 \pm 3.34$, confirming that the condition impairs quality of life. Previous studies evaluating the quality of life in patients with bullous dermatoses have included smaller groups of BP patients and demonstrated similar results. ${ }^{4,5,12,13}$ Patients struggle to

TABLE 1: Anxiety, depression and loneliness in BP patients, quantitatively compared (t-test) with healthy volunteers

\begin{tabular}{lllll}
\hline & $\begin{array}{l}\text { BP Patients } \\
(\mathbf{n}=\mathbf{5 7})\end{array}$ & $\begin{array}{l}\text { Controls } \\
(\mathbf{n = 5 7 )}\end{array}$ & t-test & p-value \\
\hline Total score UCLA scale mean \pm SD & $43.01 \pm 5.66$ & $39.12 \pm 7.79$ & 3.05 & 0.003 \\
Total score HADS-total mean \pm SD & $13.68 \pm 4.07$ & $11.85 \pm 3.84$ & 2.45 & 0.015 \\
Total score HADS-anxiety mean \pm SD & $5.91 \pm 2.30$ & $5.56 \pm 2.44$ & 0.788 & 0.43 \\
Total score HADS-depression mean \pm SD & $7.77 \pm 2.36$ & $6.42 \pm 2.09$ & 3.22 & 0.002 \\
\hline
\end{tabular}


cope with the disease in everyday life since it affects activities like walking, carrying and climbing stairs. Specific daily activities such as home management (problems with work around the house or garden), clothing (having to change or wash clothes more often) and bathing. can also be complicated. Thus, patients experience great frustration due to the passive and unfulfilling daily routine. ${ }^{14,15}$

BP's adverse effects on body self-image (feeling unclean and physically unattractive), the disease's chronic nature and the difficulty in controlling symptoms may explain the high scores recorded on the HADS-depression scale. Furthermore, pain, itching and discomfort can have a negative impact on sleep, mood and enjoyment of life, representing further cause for depression in patients. ${ }^{15}$

The results of the UCLA loneliness scale indicated that personal relationships with relatives and friends can be also impaired. Skin lesions make them feel disfigured and repulsive; consequently, patients reduce their social contact. As a result, they feel lonely and socially isolated. To the best of our knowledge, no previous studies have evaluated the incidence of depression and loneliness among BP patients.
A positive correlation was documented between depression and loneliness in BP patients. This may be attributable to restrictions on work and daily activities, patients' concerns over their appearance, the BP treatment and its probable recurrence. Proper management of depression may help patients to overcome loneliness and eventually to feel happier and more fulfilled.

The main limitation of our study is the relatively low number of patients. However, this does not detract from the importance of the results. BP had a significant impact on the quality of life and psychological status of our patients. BP management focuses mainly on the healing process rather than the patient's emotional status. Introducing quality of life evaluation into clinical practice could help clinicians to select patients who would benefit most from the addition of psychological and social support, offering patients relief from isolation and an opportunity to share common experiences.

\section{CONCLUSION}

Bullous pemphigoid had a significant impact on the quality of life and psychological status of our patients, probably due to the appearance of unattractive lesions on the skin, functional problems and disease chronicity. $\square$

\section{REFERENCES}

1. Feliciani C, Joly P, Jonkman MF, Zambruno G, Zillikens D, loannides D, et al. Management of bullous pemphigoid: the European Dermatology Forum consensus in collaboration with the European Academy of Dermatology and Venereology. Br J Dermatol. 2015;172:867-77.

2. Teixeira VB, Cabral R, Brites MM, Vieira R, Figueiredo A. Bullous pemphigoid and comorbidities: a case-control study in Portuguese patients. An Bras Dermatol. 2014;89:274-8.

3. Penha MÁ, Farat JG, Miot HA, Barraviera SR. Quality of life index in autoimmune bullous dermatosis patients. An Bras Dermatol. 2015;90:190-4.

4. Zhao CY, Murrell DF. Outcome measures for autoimmune blistering diseases. J Dermatol. 2015;42:31-6.

5. Sebaratnam DF, Hanna AM, Chee SN, Frew JW, Venugopal SS, Daniel BS, et al. Development of a quality-of-life instrument for autoimmune bullous disease: the Autoimmune Bullous Disease Quality of Life questionnaire. JAMA Dermatol. 2013;149:1186-91.

6. Tjokrowidjaja A, Daniel BS, Frew JW, Sebaratnam DF, Hanna AM, Chee S, et al. The development and validation of the treatment of autoimmune bullous disease quality of life questionnaire, a tool to measure the quality of life impacts of treatments used in patients with autoimmune blistering disease. $\mathrm{Br} \mathrm{J}$ Dermatol. 2013;169:1000-6.

7. Finlay AY, Khan GK. Dermatology Life Quality Index (DLQI)-a simple practical measure for routine clinical use. Clin Exp Dermatol. 1994;19:210-6.

8. Zigmond AS, Snaith RP. The Hospital anxiety and depression scale. Acta Psychiatr Scand. 1983;67:361-70.

9. Michopoulos I, Douzenis A, Kalkavoura C, Christodoulou C, Michalopoulou P, Kalemi G, et al. Hospital Anxiety and Depression Scale (HADS): validation in a Greek general hospital sample. Ann Gen Psychiatry. 2008;7:4.

10. Russell DW. UCLA Loneliness Scale (Version 3): Reliability, validity and factor structure. J Pers Assess. 1996;66:20-40.

11. Kafetsios, K. Adult Attachment, coping with stress and social support as predictors of mental health. Comparative results from Greece and the UK. Abstracts of the International Conference in Personal Relationships. Australia: University of Queensland; 2000. P.38-39.

12. Moon SH, Kwon HI, Park HC, Kim JE, Ko JY, Ro YS. Assessment of the Quality of Life in Autoimmune Blistering Skin Disease Patients. Korean J Dermatol. 2014; 52:402-9.

13. Sebaratnam DF, McMillan JR, Werth VP, Murrell DF. Quality of life in patients with bullous dermatoses. Clin Dermatol. 2012;30:103-7.

14. Sebaratnam DF, Frew JW, Davatchi F, Murrell DF. Quality-of-life measurement in blistering diseases. Dermatol Clin. 2012; 30:301-07.

15. Fried RG, Gupta MA, Gupta AK. Depression and skin disease. Dermatol Clin. 2005; 23: $657-64$.

\begin{tabular}{l}
\hline MAILING ADDRESS: \\
Kouris Anargyros \\
I.Dragoumi 5, \\
16121, Athens, Greece \\
2. Rimini 1 \\
12461 Xaidari, Greece. \\
E-mail: kouris2007@yahoo.com
\end{tabular}

How to cite this article: Kouris A, Platsidaki E, Christodoulou C, Armyra K, Korkoliakou P, Stefanaki C, Tsatovidou R, Rigopoulos D, Kontochristopoulos G. Quality of life, depression, anxiety and loneliness in patients with bullous pemphigoid. A case control study. An Bras Dermatol. 2016;91(5):601-3. 